\title{
Gilles Lepesant (dir.), 2018, Énergies nouvelles, territoires autonomes? Paris, Presses de l'Inalco, 296 pages.
}

Nicolas Dupuis

\section{CpenEdition}

Journals

Édition électronique

URL : http://journals.openedition.org/developpementdurable/17772

DOI : 10.4000/developpementdurable. 17772

ISSN : 1772-9971

Éditeur

Association DD\&T

\section{Référence électronique}

Nicolas Dupuis, «Gilles Lepesant (dir.), 2018, Énergies nouvelles, territoires autonomes ? Paris, Presses de l'Inalco, 296 pages. », Développement durable et territoires [En ligne], Vol. 11, n³ | Décembre 2020, mis en ligne le 20 décembre 2020, consulté le 14 janvier 2021. URL : http://journals.openedition.org/ developpementdurable/17772 ; DOI : https://doi.org/10.4000/developpementdurable.17772

Ce document a été généré automatiquement le 14 janvier 2021.

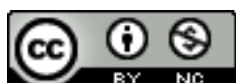

Développement Durable et Territoires est mis à disposition selon les termes de la licence Creative Commons Attribution - Pas d'Utilisation Commerciale 4.0 International. 


\section{Gilles Lepesant (dir.), 2018, Énergies nouvelles, territoires autonomes? Paris, Presses de l'Inalco, 296 pages.}

\section{Nicolas Dupuis}

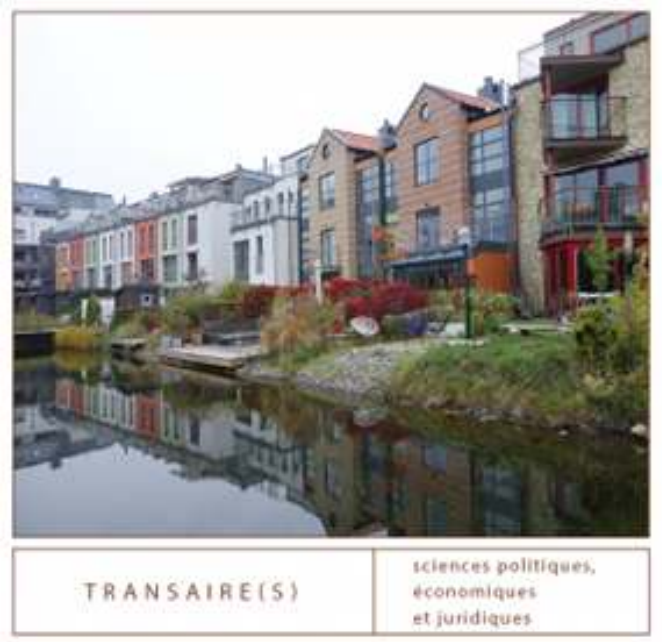

\section{ÉNERGIES NOUVELLES,}

TERRITOIRES AUTONOMES ?

Gilles Lepesant (dir.) $\frac{\text { in a Ico }}{\text { PRESSES }}$

L’ouvrage dirigé par Gilles Lepesant est composé de 8 chapitres répartis sur 232 pages. Les thématiques de la transition et de l'autonomie énergétiques dans les territoires y sont traitées en abordant des exemples à l'international. Chaque chapitre présente et analyse un territoire dans son contexte national. Les auteurs abordent avant tout les modes de gouvernance liées aux initiatives énergétiques, ainsi que les difficultés 
rencontrées. Elles proviennent généralement de conflits entre des besoins locaux, un équilibre entre les marchés et un alignement sur des réglementations nationales ou européennes.

2 Xavier Lagurgue évoque dans son chapitre la prise en compte des besoins architecturaux et sociaux pour atteindre une efficacité et une autonomie énergétiques dans le bâti. En effet, l'auteur précise que les architectes devront « dans le même temps comprendre la nature des objectifs poursuivis et s'approprier de façon explicite la notion d'énergie, l'extraire de sa dimension strictement technique et mettre en évidence les enjeux culturels dont elle relève » (p. 112). Il rappelle ainsi que l'énergie ne fait pas uniquement partie d'un processus technique, mais s'insère dans un cadre de vie. Il en va de même pour l'autonomie énergétique qu'il envisage de l'échelle de l'habitat à celle du territoire, ce qui invite à des réflexions tant individuelles que nationales. L'auteur souligne deux enjeux de gouvernance: prendre en compte les spécificités locales dans les réflexions au niveau national et évaluer la soutenabilité des projets locaux sur le long terme, afin de pouvoir envisager un déploiement à plus grande échelle.

3 C'est d'ailleurs ce que Fanny Lopez met en évidence dans le cas de la ville de Londres, au Royaume-Uni. Elle montre que la plupart des initiatives d'entreprises privées (coopératives énergétiques, réseau d'équipements interconnectés à l'échelle d'un quartier, centrales de cogénération, etc.) doivent répondre à des normes gouvernementales pour proposer des bâtiments (habitation et bureaux) à haute valeur ajoutée. Au-delà des surcoûts économiques des opérations répercutés sur les prix de vente, certains promoteurs mettent en avant une image haut de gamme de ces bâtiments pour cibler une clientèle aisée. La rentabilité économique de l'utilisation des nouveaux systèmes énergétiques n'est pas assurée, même à long terme et malgré les aides de l'État, en raison des coûts élevés de recherche et de développement, ainsi que de ceux liés à l'installation et à la maintenance des systèmes. De plus, la diminution de la consommation n'est efficace que si les usagers respectent également les conditions d'utilisation des énergies. Par exemple, certaines personnes peuvent être tentées de chauffer davantage si le coût de la consommation est réduit. Aussi, le raccordement des bâtiments aux réseaux urbains n'est pas systématique, de par l'incompatibilité de certaines technologies aux réseaux urbains existant (électricité, chaleur, etc.), ce qui limite la redistribution des surplus de production énergétique.

4 Cela tranche avec les réalisations de Malmö en Suède, décrites par Gilles Lepesant. Tout est pensé pour être synchronisé et en interaction dans une même municipalité : les besoins énergétiques en chaleur des citoyens sont assouvis par une usine de recyclage des déchets. L'auteur relève que l'économie circulaire développée entre la gestion des déchets et le réseau de chaleur à Malmö visait à contrecarrer une crise économique et industrielle locale. Cependant, la ville n'a pas vraiment été soutenue par l'État suédois et a été confrontée à la législation du pays et aux directives européennes, notamment concernant la concurrence, car l'entreprise municipale de recyclage des déchets risquait d'exercer un monopole énergétique. Malgré ces difficultés, la ville a réalisé son projet en s'appuyant parfois sur des flous réglementaires, notamment en ce qui concerne la question du monopole.

5 Nous pouvons noter que le modèle de Malmö est dépendant d'une seule solution phare orientée vers l'usine de recyclage, tandis que les initiatives proposées à Londres permettent de tester plusieurs modèles et technologies. Ces deux modèles de transition 
s'opposent donc, entre efficacité à Malmö (permise par la synchronisation forcée, mais une dépendance à un seul modèle) et une pluralité de solutions à Londres (qui permet une forte adaptation aux divers besoins, mais une faible synchronisation). À la suite de son enquête londonienne, Fanny Lopez soulève ainsi quelques questionnements qu'il reste à explorer: "La multiplication des projets et de ces micro-infrastructures constitue-t-elle une stratégie énergétique articulée? Quels sont les effets spatiaux de ces phénomènes de décentralisation énergétiques dans le tissu urbain ?» (p. 30).

6 La ville d'Aberdeen, au Royaume-Uni, a également essayé de soutenir les initiatives liées aux énergies renouvelables, sur un modèle hybride entre ceux de Londres et de Malmö, mais sous la contrainte des choix gouvernementaux. Rachel Guyet décrit ainsi que «malgré la complexité des relations entre les différents niveaux de gouvernance, les villes britanniques et écossaises investissent de plus en plus le champ de l'énergie grâce aux évolutions législatives" (p.143). Ces évolutions législatives, comme l'autorisation de vendre au niveau national le surplus d'électricité produite localement, montrent que les efforts des élus locaux et la portée des projets liés à l'énergie peuvent être soutenus par l'État. Même si Aberdeen n'est pas dans une recherche d'indépendance énergétique, elle s'engage dans une rupture avec la dépendance aux énergies fossiles et souhaite valoriser les innovations énergétiques, tout en diversifiant son économie avec les énergies renouvelables (solaire et éolienne, notamment). Cette diversification est motivée par des difficultés liées à une économie locale qui est justement dépendante d'activités gazières et pétrolières qui périclitent et des initiatives avec les énergies renouvelables confrontées à des lourdeurs administratives. L'exemple d'Aberdeen fait ainsi écho à celui de Malmö dans la réflexion énergétique pour contrer une crise économique et capitaliser sur la transition énergétique, afin de sauvegarder l'emploi dans un modèle plus vertueux pour l'environnement. Il pourrait également inspirer d'autres villes qui rencontrent des problématiques similaires et cherchent des solutions alliant environnement, énergies, économie et qualité de vie.

7 À une échelle nationale, le cas de l'Allemagne, décrit par Anaïs Guerry, montre que le fonctionnement politique et législatif même du pays entre en jeu dans la régularisation énergétique. En effet, l'Allemagne se veut exemplaire en matière d'énergie renouvelable, mais doit faire face à la pollution émise par le recours aux énergies fossiles comme le charbon pour compléter sa production énergétique durant la transition. De plus, la gestion par Land (l'équivalent de nos régions), pose également des difficultés, car tous les Lander ne s'impliquent pas de la même manière dans le défi de la transition énergétique. L'auteur observe ainsi une opposition entre libéralisation du marché et régularisation nationale. Alors que le modèle allemand donne des moyens techniques, législatifs et financiers, à la fois au niveau fédéral et au niveau régional, tous les Lander ne s'en saisissent pas de la même manière, créant ainsi des disparités au niveau national. L'auteure précise que ce modèle permet tout de même de valoriser des « initiatives privées mais aussi collectives pour lesquelles les coopératives de production d'énergie constituent le meilleur exemple, et les initiatives privées individuelles, comme l'autoconsommation » (p.100). Par ailleurs, le dialogue entre sciences et politique est facilité, donnant aux scientifiques « une mission de conseil en politique publique » (p. 66), permettant ainsi de penser la transition énergétique entre stratégie de politique environnementale et application des travaux scientifiques.

8 À l'inverse de celui proposé en Allemagne, le modèle japonais ouvre de manière beaucoup plus limitée le dialogue entre les citoyens, les collectivités et les 
représentants de l'État, et ne laisse donc que peu de place aux initiatives locales. En effet, Magali Dreyfus indique que la plupart des prises de décisions sont centralisées par le gouvernement national. Les collectivités locales n'ont que peu de marges de manœuvre pour mettre en place des initiatives. Éveillées par la catastrophe nucléaire de Fukushima en 2011, elles souhaitent un fonctionnement plus décentralisé en ce qui concerne l'énergie, mais leur élan est parfois freiné par des moyens limités qui les rendent dépendantes des aides de l'État et des partenaires privés. De plus, les enjeux énergétiques restent majoritairement pilotés par l'État japonais, contrairement au modèle allemand qui soutient davantage la décentralisation pour ces sujets. Les deux exemples, allemands et japonais, montrent bien les divergences de gestion nationale de la transition énergétique et de l'autonomisation des territoires, ainsi que les rapports parfois conflictuels entre les entités administratives.

Pour terminer, soulignons que la richesse de l'ouvrage tient de l'analyse de la gouvernance de la transition énergétique à diverses échelles territoriales. Dans sa conclusion, Gilles Lepesant relève qu'il n'y pas de modèle unique, ni de définition précise de cette transition énergétique. Il note également un paradoxe entre la libéralisation du secteur de l'énergie et les initiatives des municipalités locales, ce qui complexifie la mise en place d'un réseau énergétique coordonné. S'ajoutent à cela les difficultés de décentralisation des compétences administratives et de celles liées à des obligations réglementaires, comme le devoir d'ouverture à la concurrence, instituées par les États ou l'Union Européenne.

Si l'ouvrage permet bien une réflexion sur les modes de gouvernance, il ne fait qu'effleurer le sujet de l'implication citoyenne, ainsi que celui des motivations individuelles et collectives à propos de la transition énergétique. En effet, la mise en place de projets liés à des énergies nouvelles nécessite de prendre en compte leur acceptabilité sociale pour à la fois informer, impliquer et recueillir l'approbation des populations résidentes d'un territoire (Dupuis, 2018). L'enjeu est important car la gouvernance seule des projets n'est pas en capacité d'envisager toutes les conditions d'acceptation des installations et des usages. Une autorisation purement administrative ne garantit donc pas l'acceptation du projet, ni une implication citoyenne sur le court, ou le long terme, surtout si aucune forme de participation n'est envisagée pour informer et débattre (Le Blanc, Frère, Chambon, Grembo et Calvo-Mendieta, 2017). Les travaux présentés dans l'ouvrage dirigé par Lepesant ayant été réalisés avec précision, nous pouvons également en retenir que si l'implication citoyenne n'est que peu décrite, c'est qu'elle est, encore aujourd'hui, peu envisagée dans ce type de projets. Les auteurs appellent justement à davantage anticiper les usages pour mieux connecter les réseaux. La mise en lumière des différents modes de gouvernance et de gestion de l'énergie, à toutes les échelles, ouvre alors la voix à une réflexion plus globale entre écologie, technique, économie, politique et usages. 


\section{BIBLIOGRAPHIE}

Dupuis N., 2020, L'acceptabilité sociale de l'hydrogène et son processus de co-construction, un enjeu pour la transition énergétique dans les territoires. Thèse de doctorat, Université du Littoral Côte d'Opale, $323 \mathrm{p}$.

Le Blanc A., Frère S., Chambon M., Grembo N. et Calvo-Mendieta I., 2017, « L'établissement du PPRT, entre rigidités institutionnelles et arrangements locaux », In Frère S. et Flanquart H. (dir.), La ville et ses risques : habiter Dunkerque, Villeneuve d'Ascq, Presses Universitaires du Septentrion, p. 177-204.

\section{AUTEUR}

\section{NICOLAS DUPUIS}

Nicolas Dupuis est psychologue social et docteur en Sciences Humaines et Humanités (spécialité en aménagement de l'espace et urbanisme). Sa recherche doctorale et ses autres expériences professionnelles en libéral lui confèrent une spécialisation concernant l'acceptabilité sociale des innovations énergétiques, plus précisément dans les domaines de la mobilité et de l'hydrogène. 